\title{
The effects of carbon electrode surface properties on bacteria attachment and start up time of microbial fuel cells
}

C. Santoro ${ }^{a, b, 1}$, M. Guilizzoni ${ }^{c, *, 1}$, J.P. Correa Baena ${ }^{a, b}$, U. Pasaogullari ${ }^{b, d}$, A. Casalegno ${ }^{~}$, B. Li ${ }^{a, b, *}$, S. Babanova ${ }^{e}, \mathrm{~K}$. Artyushkova $^{e}$, P. Atanassov ${ }^{e}$

a Department of Civil and Environmental Engineering, University of Connecticut, 261 Glenbrook Rd., Storrs, CT 06269, USA

${ }^{\mathrm{b}}$ Center for Clean Energy Engineering, University of Connecticut, 44 Weaver Rd., Storrs, CT 06269, USA

c Department of Energy, Politecnico di Milano, Via Lambruschini 4, 20156 Milan, Italy

d Department of Mechanical Engineering, University of Connecticut, 191 Auditorium Rd., Storrs, CT 06269, USA

e Department of Chemical \& Nuclear Engineering, Center for Emerging Energy Technologies, University of New Mexico, Albuquerque, NM 87131, USA

\section{A R T I C L E I N F O}

Article history:

Received 15 June 2013

Accepted 21 September 2013

Available online 1 October 2013

\begin{abstract}
A B S T R A C T
Surface roughness, porosity and contact angles of different carbon paper materials (TORAY paper with PTFE from $0 \%$ to $60 \%$ of and SGL paper with $0 \%$ and $20 \%$ of PTFE) suitable as electrodes in microbial fuel cells were investigated. The changes of contact angle between dry and clean anode surfaces and the ones after exposure to wastewater were measured using different liquids (pure water and sodium acetate solutions). The results showed that bacterial attachment to the carbon papers caused a significant decrease in the contact angle, shifting the surface property from highly hydrophobic to slightly hydrophobic or even hydrophilic. The quantity of biofilm attached on the anode surface decreased with the increase in PTFE content. Positive correlation between dry biomass content and the amount of pores at the small scale $(5-10 \mu \mathrm{m})$ was observed. The start up time of MFCs was shortened by using the carbon anodes without PTFE or with low PTFE content ( $<20 \mathrm{wt} \%$ ), probably due to the easier biofilm attachment on the surface. On the contrary, the carbon anodes with high PTFE contents had longer start up time. After several cycles of MFC operation, the performances became similar (20-30 mV of differences) regardless of the carbon anode used.
\end{abstract}

(C) 2013 Elsevier Ltd. All rights reserved.

\section{Introduction}

A microbial fuel cell (MFC) is a novel device capable of generating electricity from organic compounds in wastewater. This makes MFCs a promising biotechnology for wastewater treatment and clean energy production. Theoretically, MFCs could give a substantial contribution to solve wastewater contamination and aid in energy sustainability problems. Regrettably, energy production and organics degradation in MFCs are still low. In the past decade, several important improvements of MFC configurations were conducted to increase the power generation from MFCs [1], and one of the most important

\footnotetext{
* Corresponding authors. Addresses: Department of Energy, Politecnico di Milano, Via Lambruschini 4, 20156 Milan, Italy (M. Guilizzoni), Department of Civil and Environmental Engineering, University of Connecticut, 261 Glenbrook Rd., Storrs, CT 06269, USA (B. Li).

E-mail addresses: manfredo.guilizzoni@polimi.it (M. Guilizzoni), baikun@engr.uconn.edu (B. Li).

1 These authors have contributed equally to this work. 0008-6223/\$ - see front matter @ 2013 Elsevier Ltd. All rights reserved. http://dx.doi.org/10.1016/j.carbon.2013.09.071
} 
was the removal of the solid electrolyte (polymeric membrane) in the single chamber MFC (SCMFC) systems [1], where anodes are immersed in wastewater and cathodes are exposed to air (oxygen).

There are different requirements for anode and cathode materials. Anode electrodes are usually composed of hydrophilic carbon based materials with high surface area and high electrical conductivity [1,2]. Particularly, the high surface area allows high extent of bacteria attachment and increases the surface area available for electron exchange between bacteria and the electrode $[2,3]$. The surface repulsion forces should be low in order to promote biofilm settlement and bonding $[1,3,4]$. Anodes should also have high electronic conductivity in order to reduce the ohmic resistance of the electron flow in MFCs. Cathodes are characterized with high electron conductivity in order to distribute the electrons generated from the anode, high mechanical strength to support the cathode structure, and good porosity and hydrophobicity to provide oxygen diffusion to the catalytic (e.g. platinum) sites [5]. The open pores of the cathode allow oxygen transport from the atmosphere to the catalyst for oxygen reduction reaction (ORR). In order to prevent "soaking" of the cathode with electrolyte and maintain the oxygen transport through the electrode structure, the cathode material is usually treated with a wetproofing agent, e.g. polytetrafluoroethylene (PTFE) [5], which makes the material hydrophobic (i.e. contact angle with water higher than $90^{\circ}$ ) and thus increases oxygen diffusion [5]. The PTFE results in a reduction in the surface conductivity but improves the ORR on the electrode. Unlike the anode, the biofilm attachment on the cathode surface is not desired, since the biofilm will cover the catalyst and hinder the ORR [6].

The wettability of anode and cathode directly affect the MFC performance, as it significantly influences the biofilm growth on the anode, the electrons transfer from bacteria to the anode surface, and the oxygen transport throughout the cathodes for ORR. It is therefore critical to characterize the electrode wettability in order to select electrode materials appropriate for MFC applications. The wettability of unsmooth surfaces mainly depends on the surface wettability, roughness and porosity. One of the most common methods for analyzing the hydrophobicity/hydrophilicity of a surface is the measurement of the contact angle, i.e. the angle which a drop of liquid deposited on the surface forms with the latter at the line where the two and the surrounding medium are all in contact (the so-called triple line). The contact angle can be evaluated in static or dynamic (drop growth/advancement or shrinking/recession) conditions. The static contact angle can be theoretically (i.e. on ideal surfaces) linked to the interfacial energies of the phases (air, liquid and solid) in contact by means of the Young equation [7]:

$\cos \theta=\frac{\sigma_{\mathrm{SV}}-\sigma_{\mathrm{SL}}}{\sigma_{\mathrm{LV}}}$

where $\sigma_{S V}$ is the interface energy between the solid and the vapour, $\sigma_{\mathrm{SL}}$ is the interface energy between the solid and the liquid, $\sigma_{L V}$ is the interface energy between the liquid and the vapour (also named surface tension).

On non-ideal surfaces, e.g. surfaces which are chemically not homogeneous or curved or rough, the contact angle may be different from point to point along the triple line. Moreover, if the surface is rough, only the apparent contact angle (i.e. the angle that the drop contour forms with an ideal horizontal line cutting the base surface at the contact points) is accessible to conventional optical experiments [8]. Despite such limitations and some studies questioning the effective validity of such "external" contact angle for the prediction of the in-pore behaviour of the porous materials [9], the contact angle is still a widely used "lumped parameter" which provides valuable information about the macroscopic characteristics of the surfaces. In the present study, it was analyzed in synergy with two other surface parameters: roughness and porosity.

Although there are several studies about biofilm growth on anodes and cathodes in MFCs $[1-4,10,11]$, there has been no effort to correlate the biofilm formation with the surface characteristics of carbon paper materials before and during biofilm development period, which hinders the thorough understanding of the changes of the electrode properties during the start-up stage of MFCs. In this study, the initial surface properties, in particular the surfaces roughness, porosity and wettability (in terms of contact angle measured using the sessile drop technique) were investigated in "clean" conditions. A possible explanation of the relationship between hydrophobic pre-treatment (PTFE\%) and contact angle was also presented. Moreover, the contact angles of carbon paper materials were investigated after biofilm development to show the changes in surface characteristics due to the interaction between material and bacteria. Several types of carbon papers suitable as anode electrode support for MFC application were compared. The effects of carbon paper structure and hydrophobic pre-treatments on surface characteristics were examined. Finally, those carbon papers were investigated in the start-up stage of SCMFCs in order to correlate the materials surface properties with biofilm formation and current production in MFCs.

\section{Materials and methods}

\subsection{Electrode materials}

Two types of carbon paper materials (TORAY H090 and SGL S35, Ion Power) were studied in this experiment. Particularly, TORAY H090 series with different PTFE content (H090-0, 0 wt\%; H090-20, 20 wt\%; H090-40, 40 wt\%; H090-60, 60 wt\%) and SGL S35 series with PTFE content of 0 and $20 \%$ (S35-0, 0 wt\%; S35-20, 20 wt\%) were investigated (Table 1). The carbon materials were composed from fibers with diameters ranging between 6 and $10 \mu \mathrm{m}$ and lengths between 3 and $20 \mathrm{~mm}$. These commercial materials were specifically selected in order to investigate the effect of the PTFE content on the surface characteristics. Surface hydrophobicity was measured under two conditions: first, the carbon papers were in "clean" and dry condition; second, carbon papers were completely immersed in primary wastewater taken from the Nosedo Wastewater treatment plant (Milan, Italy) for 2 weeks, with biofilms growing on cathode surfaces. Three types of liquids were used for the contact angle measurement in "clean" and dry conditions: distilled water, water with $1 \mathrm{gL}^{-1}$ of sodium acetate (NaOAc), and water with $3 \mathrm{~g} \mathrm{~L}^{-1}$ of NaOAc. The last two solutions represented the organic substrate concentrations of wastewater commonly used in MFCs. 
Table 1 - Characteristics of the carbon papers investigated in this study.

\begin{tabular}{lcccc} 
Commercial company & Commercial name & Hydrophobic treatment (wt\% PTFE) & Thickness $(\mu \mathrm{m})$ & Nomenclature \\
\hline TORAY & H090 & 0 & 280 & H090-0 \\
TORAY & H090 & 20 & 280 & H090-20 \\
TORAY & H090 & 40 & 280 & H090-40 \\
TORAY & H090 & 60 & 280 & H090-60 \\
SGL & S35 AA & 0 & 300 & S35-0 \\
SGL & S35 DA & 20 & 300 & S35-20 \\
\hline
\end{tabular}

\subsection{Determination of the surface roughness and porosity}

Digital image processing of SEM images, including roughness and texture analysis was done with the in-house written GUI [12] in the Matlab ${ }^{\oplus}$ Image Processing Toolbox [13]. Five replicate SEM images at $5 \mathrm{kV}$ and 5000× magnification from various areas on the sample were acquired. The five SEM images for each sample were processed and the resulting statistical parameters were averaged. During SEM acquisition, all experimental parameters, i.e. voltage, magnification, current and gain, were kept constant from sample to sample to ensure that intensity variability in images is caused only by the sample variability and not by instrumental factors. The utilization of digital image processing to determine the morphology from microscopic images has been previously presented [14].

\subsection{Contact angle measurement}

Apparent contact angles (as the investigated surfaces were characterized by a significant surface roughness) were measured with the sessile drop technique (Fig. 1), using "as placed drops" [15], i.e. gently deposited drops on a fixed surface with no vibration or other disturbances. The sessile drop technique is probably the most used approach for contact angle evaluation [16]. A high precision metering pump with suitable syringes (Hamilton) was used to generate drops of controlled volume for contact angle measurement. A $800 \mathrm{~W}$ lamp equipped with a diffuser provided the lighting necessary for the photographic acquisitions, which were taken using a SLR digital camera Nikon D90 with a AFS $60 \mathrm{~mm}$ F2.8 Macro lens (Fig. 1a). Side shots of the deposited drop were acquired and transferred to a computer, and the images were analyzed using home-made software within the Matlab ${ }^{\varpi}$ programming environment. A photograph of the side view of the drop and another of the background were taken using the camera (Fig. 1b). These two images were registered to get the best possible alignment between the two, and the Sobel operator [17] was then applied to the pixel-wise difference between these two images. Holes and objects touching the image borders were removed and the extracted drop contour was smoothed using a cubic smoothing spline.

For surfaces where the surface texture is fairly uniform and isotropic, the Laplace-Young equation [18] may still give a good approximation of the drop contour. It reads:

$\Delta \mathrm{P}_{\text {apex }}+\Delta \rho g \mathrm{y}=\sigma_{\mathrm{LV}}\left(\frac{1}{\mathrm{R}_{1}}+\frac{1}{\mathrm{R}_{2}}\right)$

where $\Delta P_{\text {apex }}$ is the pressure jump at the drop apex, $y$ is the vertical axis centered in the drop apex and with downward direction, $R_{1}$ and $R_{2}$ are the principal curvature radii. In these cases the maximum radius $R$ of the drop and the difference between the height of the apex and of the maximum diameter, $Z$ were calculated from image analysis. A biunique relation exists between the ratio $R / Z$ and the Eötvös number:

Eo $=\frac{\Delta \rho g R_{\text {apex }}^{2}}{\sigma_{\mathrm{LV}}}$

(where $\Delta \rho$ is the density difference between the densities of the drop liquid and of the surrounding atmosphere, $g$ is the modulus of the gravity vector, $R_{\text {apex }}$ is the curvature radius at the drop apex), so that the Eo of the drop could be calculated from the first [19], with no need to determine the curvature radius at the drop apex. Once Eo was obtained, the corresponding ideal drop profile was calculated using the basic finite difference (centred form) integration of the axisymmetric Laplace-Young equation [20].

The baseline, i.e. the contour of the base surface, was extracted from the images too. For highly hydrophobic surfaces or rough surfaces the latter step may require manual intervention by the operator due to the sensitivity of the results to errors in the baseline identification. The use of high quality, high resolution colour images from the SLR camera with macro lens allowed to identify the baseline and the contact points with good accuracy also on traditionally "problematic" surfaces as textiles [21,22]. Afterwards, contact angle could be then easily determined at the intersection between the calculated drop profile and the baseline. When the surface proved to be too inhomogeneous or rough to use the Laplace-Young model, the drop contour extracted from the images was directly fitted with smoothing splines [23] and the contact angle was then calculated by third-degree polynomial fitting of the left and right extreme parts of the final contour [24].

The validation of this technique was conducted first on computer-generated drop images [25] and then on real drop images on smooth surfaces: the mean absolute error (MAE) for the drops with contact angles smaller than $160^{\circ}$ was only $0.8^{\circ}$, and became $3.7^{\circ}$ for contact angles larger than $160^{\circ}$. This indicated that the sessile drop technique with image analysis used in this study was sufficiently accurate for the scopes of this work.

\subsection{Determination of attached biomass}

The change of the carbon paper "wet" weight was used as an indirect method representing the amount of bacteria attached to the electrode surface over time and the change in carbon paper wettability. After the carbon papers were immersed in wastewater for 2 weeks, the total mass and 


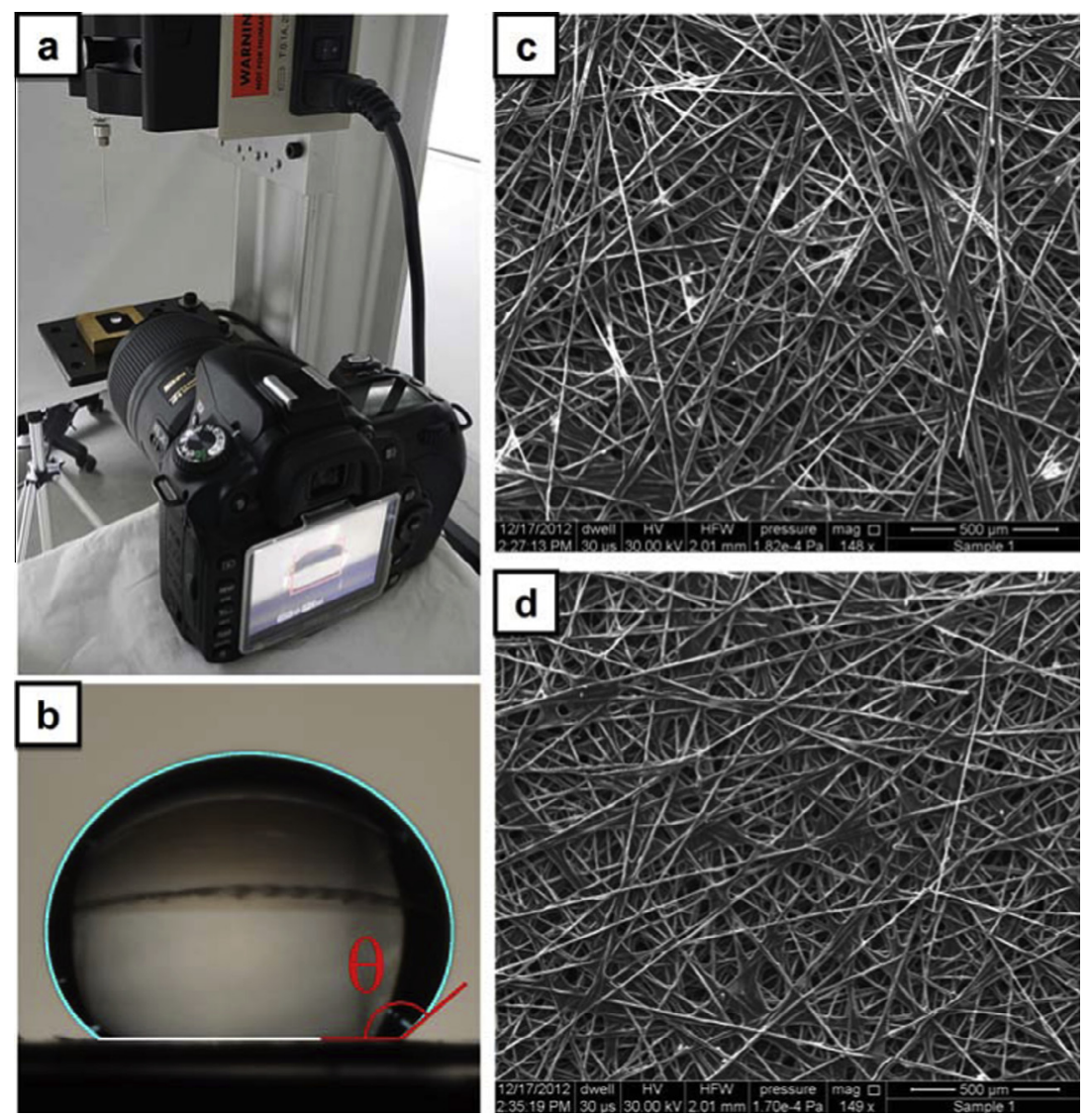

Fig. 1 - Experimental set up (a); extraction of the drop contour and contact angle measurement (b); SEM images of a H090-20 sample (c) and of a H090-60 sample (d) in dry and clean conditions. (A colour version of this figure can be viewed online.)

the "dry" mass (i.e. after they were dried to a constant mass) of the carbon papers were measured using a high precision and accuracy balance (Mettler Toledo PR503, $0.001 \mathrm{~g}$ resolution). The difference of the "dry" paper mass before and after the biofilm development was used as a representative of the attached biomass amount. The bacteria surface coverage and penetration through the fibers were elucidated using SEM images.

\subsection{Microbial fuel cell design and operation}

Membraneless single chamber microbial fuel cells (SCMFC) with air-cathode were used in this study and operated in a batch-mode. The anodic chamber had an empty volume of $130 \mathrm{ml}$ as previously described [26]. The anode materials (carbon papers) were completely immersed in the electrolyte (raw wastewater taken from a local wastewater treatment plant with $1 \mathrm{gL}^{-1}$ of additional sodium acetate $\mathrm{NaOAc}$ as easily degradable organic compound). Anode and cathode were both in contact with the same electrolyte in the membraneless SCMFC configuration. The carbon papers investigated were used as an anode electrode with no additional catalyst and with projected area of $5 \mathrm{~cm}^{2}$. The cathode was based on a three-layer structure with carbon cloth (wet proofing $30 \mathrm{wt} \%$ PTFE, Fuel Cell Earth) as electron collector facing the air, a micro porous layer (MPL), mainly composed by carbon black powder and PTFE, coated on the carbon cloth [27] and a cata- lyst layer facing directly the electrolyte. The catalyst added onto the cathode surface was platinum (Pt) with a loading of $0.25 \mathrm{mg} \mathrm{Pt} \mathrm{cm}^{-2}$ as previously described [28]. The cathode had a project area of $3.5 \mathrm{~cm}^{2}$. Anode and cathode were connected with an external resistance of $1000 \Omega$ and the voltage output was recorded every $30 \mathrm{~min}$ by a data logging system (Keithley 2700) connected to a computer. The electrolyte was refreshed after 4 days when the voltage decreased rapidly. For each carbon anode material tested, the SCMFCs were operated in parallel experiments.

\section{Results and discussion}

\subsection{Surface roughness and porosity}

A typical surface displays roughness due to finer irregularities (high-frequency component of images) overlaid over more widely spaced repetitive deviations usually called waviness (low-frequency component of images). It is vital to separate and evaluate waviness and roughness parameters from images in order to separate roughness and porosity at different scales of morphology. For this purpose, a highpass filter was applied to remove low-frequency component and a low-pass filter to remove high-frequency component from images, thus producing roughness and waviness image components, respectively. Roughness parameters (average roughness $R_{a}$ and skewness of the roughness profile $R_{s k}$ ) 
were calculated from the two frequency image components separately, on scales between 100-300 $\mu \mathrm{m},\left(R_{a}^{L}\right.$ and $\left.R_{s k}^{L}\right)$ and between $5-10 \mu \mathrm{m}\left(R_{a}^{H}\right.$ and $\left.R_{s k}^{H}\right)$ respectively. $R_{a}$ the arithmetic mean of the absolute values of the surface departure from the mean plane, $R_{s k}$ points towards domination of valleys (pores) or peaks in the micrograph, thus being a very important parameter for the analyses in this work.

The results of the carbon materials roughness and skewness or porosity are represented on Fig. 2. It was observed that for increasing amounts of PTFE, the roughness of the surface also increased at both macro and micro-scales (Fig. 2a and c). Skewness at low frequency scale increased slightly as the amount of PTFE increased, pointing to the fact that amount of pores on scale of $100-300 \mu \mathrm{m}$ increased (Fig. 2b). At the same time, the amount of pores at micro scales - from 5 to $10 \mu \mathrm{m}$ - decreased at increasing amount of PTFE (Fig. 2c). Therefore, the overall roughness on all scales rised with the amount of PTFE on the carbon papers. This was accompanied by growth of large pores, which might contribute to the increase of the overall roughness. However, a very important outcome of the digital image processing and separation of scales using filtering was that the amount of small pores in the overall rougher surface of PTFE decreased as\% of PTFE increased (Fig. 2d). The decrease of the amount of small pores led to a decrease of the overall surface area, from one side, and to a decrease of the number of pores available for bacteria attachment. Taking into account the bacteria size $(\sim 1 \mu \mathrm{m})$, it was expected that the pores preferred by bacteria were exactly in the small pore range $(5-10 \mu \mathrm{m})$. Hence, it can be hypothesized that the decrease of the skewness at the low frequency scale could cause a decrease of the amount of bacteria attached and could lead to the generation of lower current densities in MFCs. This assumption was confirmed afterwards (see Sections. 3.4 and 3.6).

\subsection{Contact angle of carbon papers in dry and "clean"} conditions

The surface hydrophobicity of the clean and dry carbon papers (TORAY and SGL) was tested with three types of liquid (pure water, $1 \mathrm{~g} \mathrm{~L}^{-1} \mathrm{NaOAc}$ solution, and $3 \mathrm{~g} \mathrm{~L}^{-1} \mathrm{NaOAc}$ solution). A light variability of contact angles was observed on each carbon surface tested (Fig. 3). Robust statistics was explored for data processing due to the insensitivity of this statistics to the presence of outliers. The median values were used as a representative of the data set and the normalized median of absolute deviation (MADN) as an equivalent of standard deviation.

The TORAY carbon papers without PTFE treatment (H0900 ) had a median contact angle of $151 \pm 3^{\circ}$ with pure water (Fig. 3a), which was consistent with previous analysis on similar surfaces [29]. Looking at the whole set of data, it was observed that the contact angle was approximately the same for the TORAY papers with and without PTFE treatment, from $135^{\circ}$ to $155^{\circ}$. (Fig. 3a). Fig. 3 represents the data using "box and whisker plot", where the median is the line in the box, the box represents the lower and the upper quartile and the tails are the minimum and the maximum values. The TORAY carbon papers investigated in this study were very hydrophobic close to the superhydrophobicity (above $150^{\circ}$ ). Previous literature showed that the effect of surface roughness on the contact angle of PTFE surfaces is complex [30], but generally, PTFE content and surface roughness have both a positive effect on the material hydrophobicity $[29,31]$. In this study, no clear trend was observed between the contact angle and the PTFE content, although the roughness at macro-scale and micro-scale has been found to increase with the PTFE content (Fig. 2a and c). This confirms the manifold nature of the wetting on textiles, which is a function both of chemistry and of their peculiar morphology. A possible explanation may be
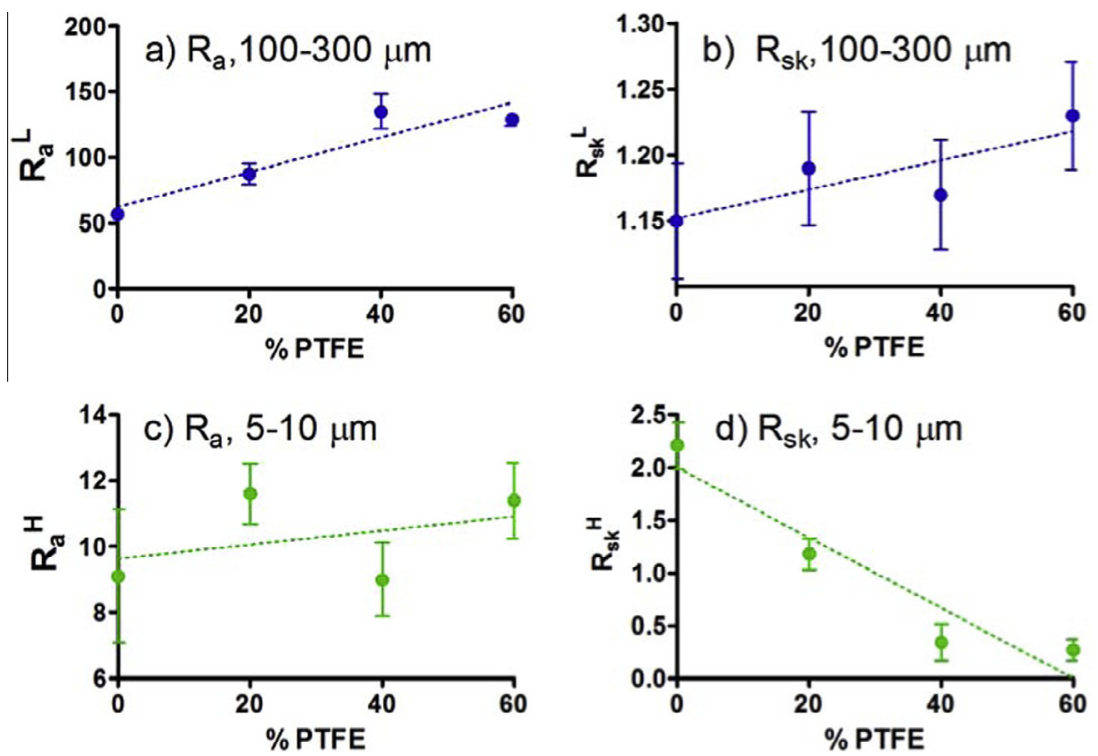

Fig. 2 - Statistical parameters calculated from the SEM images of carbon papers as a function of PTFE (wt\%), (a) low frequency roughness, (b) low frequency skewness, (c) high frequency roughness and (d) high frequency skewness. (A colour version of this figure can be viewed online.) 


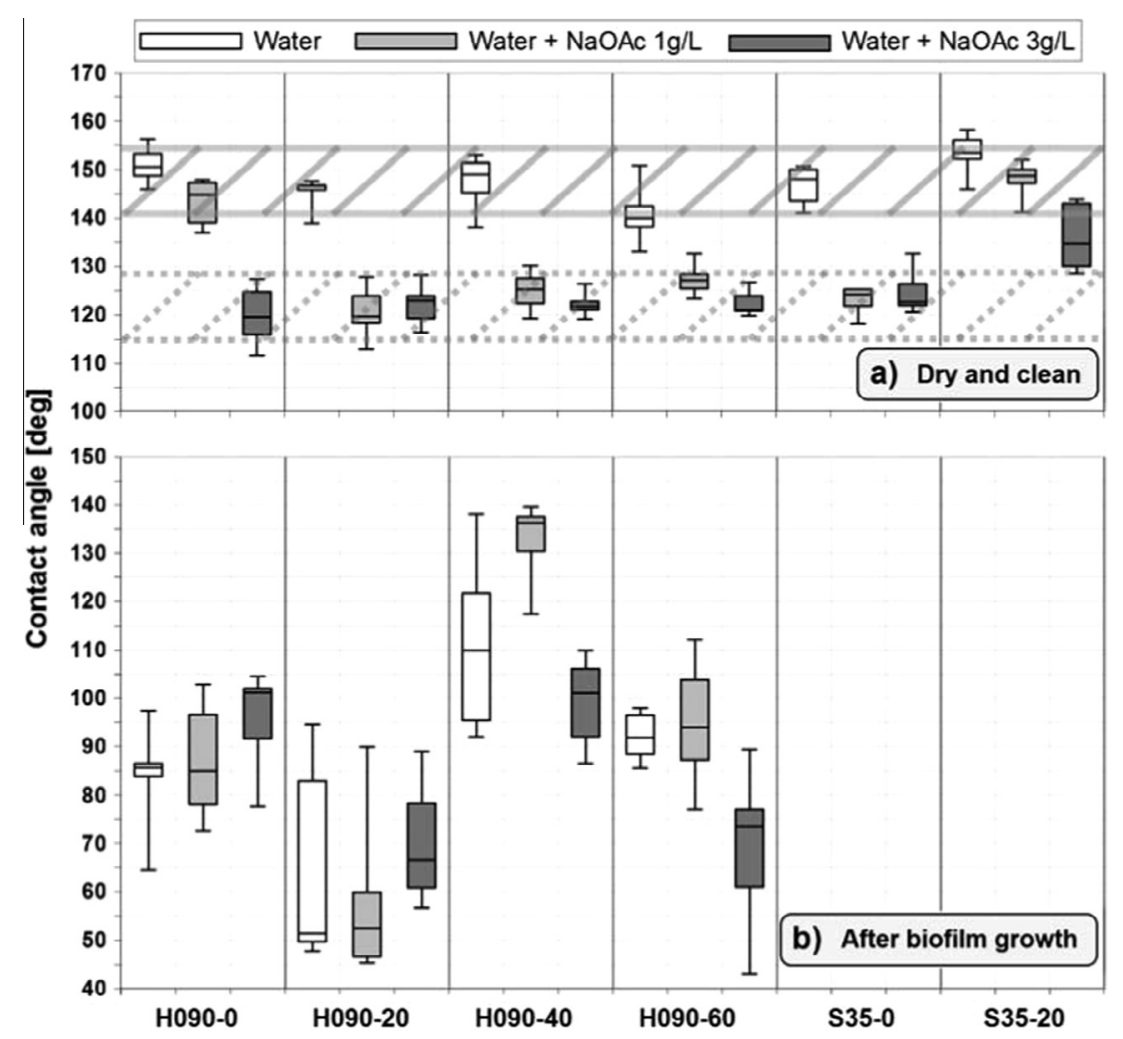

Fig. 3 - Contact angles of drops of pure water, of $1 \mathrm{~g} \mathrm{~L}^{-1}$ and $3 \mathrm{~g} \mathrm{~L}^{-1}$ of NaOAc solutions on dry and clean carbon papers samples (a) and on the same samples after biofilm growth (b).

that the higher contact angle on H090-0 was caused by the pinning effects of the three-phase line on the edges of the surface texture [32] or to the protrudent fibers on which the drop remained lifted from the surface [33]. This could be confirmed by the higher quantity of small pores at the low microns scales for H090-0 sample (Fig. 2d). At the contrary, higher quantity of larger pores $(100-300 \mu \mathrm{m})$ was found with the increase in PTFE content contributing to the high superficial roughness.

Dynamic contact angle analysis was not performed in this study, because literature information [34] and previous tests $[21,22]$ evidenced that at ambient temperature the contact angle hysteresis on such surfaces is very small, comparable or even lower than the experimental uncertainty of the static contact angle. Consequently, the results from the dynamic contact angle measurements would be of a little significance and reliability for this study. Such a small value of the contact angle hysteresis should be another confirmation of the fact that the wetting regime and the roughness effect on the clean carbon papers is in general Cassie-Baxter type [35], with the drop standing on the surface edges, without filling the grooves, as a sort of "fakir" [36]. In contrast, after biofilm growth the wetting regime was very likely to be of the Wenzel type [37], with the drop liquid filling the microgrooves on the surface.

The actual contact angle on the PTFE-treated surfaces (140-151 $)$ was higher than the contact angle on a pure PTFE smooth surface $\left(110^{\circ}\right)$ [38], which indicated a positive effect of the surface texture on hydrophobicity. When organic substrate solution ( 1 and $3 \mathrm{~g} \mathrm{~L}^{-1} \mathrm{NaOAc}$ solutions) was used, the contact angle decreased by $\sim 20^{\circ}$ compared with the same TORAY papers in water, indicating that the composition of the drop and its contents of organic substrates increased the surface wetting.

For SGL carbon papers, the contact angle increased from S35-0 (no PTFE treatment, $148 \pm 4^{\circ}$ ) to S35-20 (20 wt\% PTFE treatment, $153 \pm 4^{\circ}$ ) (Fig. 3a). On the contrary, the change in the drop composition from pure water to organic liquid (NaOAc solution) led to a steep and substantial decrease (more than $20^{\circ}$ ) in the contact angle on the S35-0 surface, similar to TORAY H090-20 and H090-40 papers. On the S35-20 surface, the effect was still significant, but more progressive with the solution concentration (similar to the H090-0 and H090-60 cases). Furthermore, the relatively low standard deviation of the measured values (MADN/standard deviation less than $4^{\circ}$ ) for both TORAY and SGL carbon papers confirmed the good reproducibility of the measurements, which indicated an equally good uniformity of the materials tested in dry and clean conditions.

\subsection{Contact angle of carbon papers after biofilm growth}

After the carbon papers were immersed in raw wastewater for 2 weeks, the materials changed their hydrophobic characteristics (Fig. 3b). The S35-0 and S35-20 became completely hydrophilic, with the drop immediately absorbed by the surface (making the contact angle measurement impossible). The surface hydrophobicity of the other materials also decreased substantially and became significantly more hydrophilic. This indicated that the biofilms formed showed 
hydrophilic characteristics. The variability in the contact angles on the tested materials became large (MADN/standard deviation around $13^{\circ}$ ) as a consequence of the uneven bacteria attachment to the surface, so that the originally hydrophobic surface was non-uniformly covered by the biofilms. There was no clear trend in contact angles using water or NaOAc solutions, which might be the result of the uneven growth of biofilms on carbon paper surfaces after 2 weeks.

There was also a significant variation among the contact angles on each single surface after biofilm growth, which was demonstrated when the same solution was deposited onto two different locations of the same surface (Fig. 4a). The contact angle observable on the left side of the side view of the drop was often different from the one on the right (Fig. 4b and c). For example, a liquid drop placed on H090-0 showed hydrophilic characteristics (contact angle $<90^{\circ}$ ) on the left side of the drop while it showed hydrophobic characteristics (contact angle $>90^{\circ}$ ) on the right side (Fig. $4 \mathrm{~b}$ ). The opposite scenario was noticed for a liquid drop placed on H090-60 (Fig. 4c). Such variability was responsible for the high standard deviation in the measured contact angles. The behaviour of the drop surface and of the triple line became complex (Fig. 4d, e and f), resulting in contact angles changing from hydrophilic to hydrophobic between adjacent points along the triple line and a non-Laplacian drop shape.

\subsection{Biofilm formation on TORAY carbon papers}

Biofilm attachment and wettability changes of the TORAY carbon paper materials were determined quantitatively by measuring the change in the "wet" weight over time (Fig. 5a). As it can be seen, the change in "wet" weight of all the TORAY carbon papers had a specific trend that can be divided in three sections as a function of the slope. In the first section, there was no significant change of carbon paper weight; in the second section a notable increase in weight was detected and the third section was characterised with low variation, having a plateau.

The duration of the first section prolonged with the increase in PTFE content (Fig. 5a). The results shows that the first section ended after about 1 day for the carbon paper with no PTFE treatment (H090-0), after about 2 days for the one with 20 wt\% PTFE (H-090-20), about 3 days for the one with 40 wt\% PTFE (H090-40) and the longer time was reached by the highest PTFE (60 wt\%) treatment investigated (about 5 days). The observed trend might be explained by the higher repulsion to water due to the higher PTFE content that resist water contact and consequently lower bacteria attachment. The sudden change in weight recorded (Fig. 5a), named as second section, could be probably due to the lost in water resistance over time mainly due to the surface bio-fouling. The abatement in water resistance corresponded well with the contact angle results (as shown in Fig. 3b): the increase in wettability certainly enhanced the bacteria attachment on the surface, both due to the greater contact between solution and to the possible change in wetting regime (likely Wenzel type), with the consequent filling of surface microgrooves with liquid. The slope of the second section was also different and reversely proportional to the PTFE content. The third section was characterized by a low change in "wet" weight most
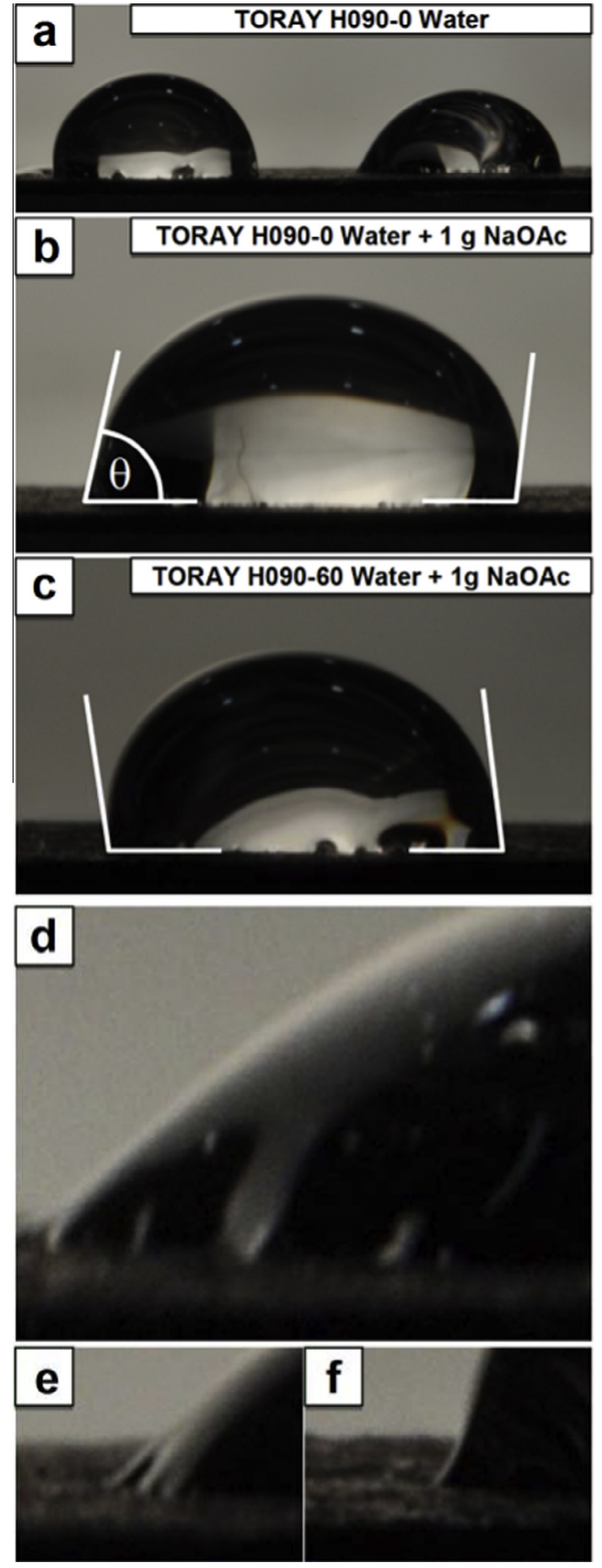

Fig. 4 - Carbon papers with biofilm after 2-weeks immersion in wastewater: two drops of the same volume deposited onto two different points on the H090-0 surface (a); the "hydrophobic" and "hydrophilic" contact angles in a single drop on the H090-0 surface (b) and on the H090-60 surface (c). Complex behaviour of the triple line and of the drop surface after biofilm growth (d, e, and f). (A colour version of this figure can be viewed online.)

probably due to the complete loss in hydrophobicity (the carbon paper cannot adsorb any additional water) and the biofilm development. 

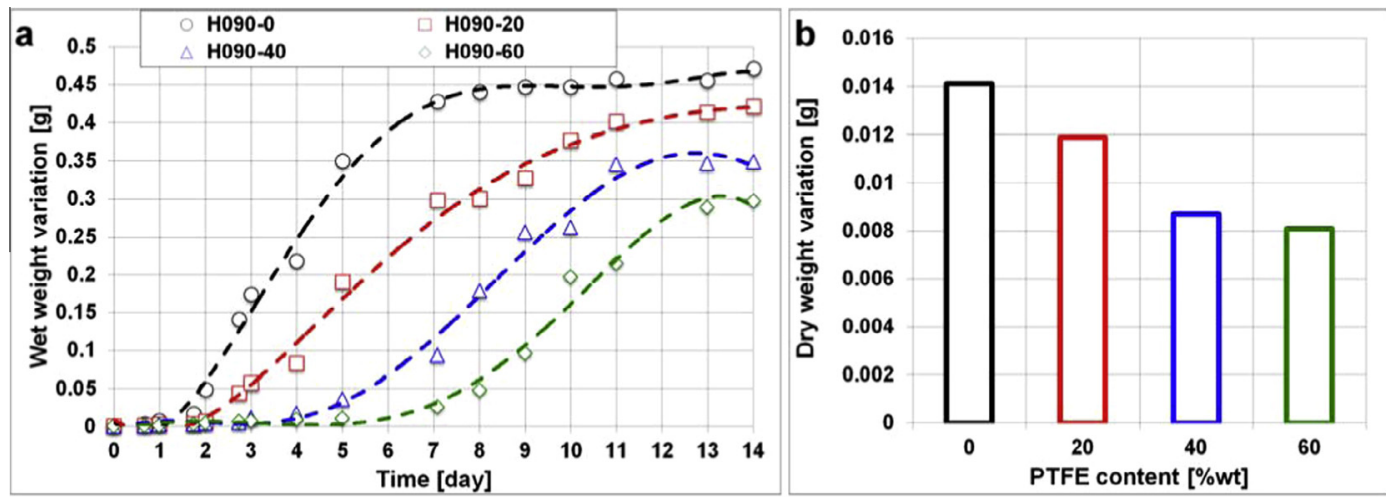

Fig. 5 - "Wet" weight variation trend over 14 days (a) and "dry" weight variation after 14 days of the TORAY carbon paper with different PTFE content. (A colour version of this figure can be viewed online.)

After 14 days the TORAY carbon papers with the developed biofilm were dried and the variation in the "dry" weight for the different papers were determined as an equivalent of the dry biomass attached (Fig. 5b). The amount of bacteria attached on the surface decreased substantially with the increase in PTFE content confirming that the decrease in the micro-scale porosity lowers significantly the biofilm attachment on the surface. A positive correlation between the high frequency skewness reflecting amount of pores at scale between 5 and $10 \mu \mathrm{m}$ versus "dry" weight variation was found (Fig. 6). Carbon papers with higher amounts of small pores presented a beneficial surface for biofilm attachment and growth.

3.5. Proposed dynamics for biofilm formation and development

The variation trend of the "wet" weight and the subsequent division in three principal sections could be explained by applying the principles of the nucleation phenomena $[39,40]$ and a possible dynamics for the biofilm formation and development on the carbon surface were hypothesized (Fig. 7). Most likely, for hydrophilic surfaces the instantaneous nucleation principle was applicable and in the case of biofilm formation it could be called "instantaneous bacterial attachment". In this early stage, the instantaneous bacterial attachment was when most of the planktonic bacterial cells simultaneously immobilised on the surface, grew fast,

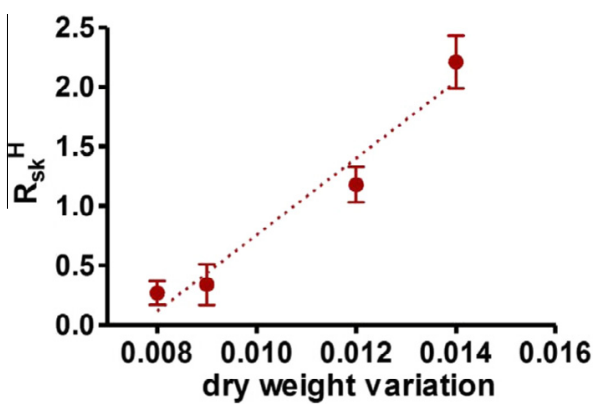

Fig. $6-R_{s k}^{H}$ extracted from the SEM images of PTFE paper versus "dry" weight variation. (A colour version of this figure can be viewed online.) proliferated and thus formed colonies that further increased in size and overlap, covering the whole surface (Fig. 7a). For hydrophobic surfaces, the progressive nucleation principle is more applicable. "Progressive bacterial attachment" is when only a number of bacteria attach to the surface, grow, proliferate and form colonies. During this process, due to the decrease of the surface hydrophobicity, new bacteria immobilized on the surface and started new "younger" colonies (Fig. 7b).

The shape of the "wet" weight variation trend over time (Fig. 5a) indicated biofilm formation through progressive bacterial attachment (Fig. 7c). The first section is where bacteria attached just in specific sites, probably in the less hydrophobic sites with the lowest possible consumption of energy and where favourable conditions were available (Fig. 7b). In the second section bacteria attached grew, developed and increased the size of each colony (Fig. 7b). When the residual hydrophobicity was completely lost (third section), new bacteria attached to the surface and started new colonies (Fig. 7b).

The confirmation that bacteria did not spread out uniformly but attached in random sections of the material can be seen on Fig. 8. This phenomenon could be caused by the intrinsic preference of the bacteria to colonize the less hydrophobic surface or the less hydrophobic parts of a surface [41].

\subsection{Start up period of SCMFCs with different carbon anodes}

The start up stage of SCMFCs with different carbon anodes materials clearly showed the effects of carbon paper surface properties on SCMFC performance. The content of PTFE in the carbon materials influenced the start up of SCMFCs (Fig. 9). Particularly, the start up was faster for the carbon papers without PTFE treatment or with low PTFE content (20 wt\%), which was the result of the easier biofilm formation on these materials with respect to the ones with higher PTFE contents (with less small pores). The increase of PTFE contents in carbon materials slowed down the start up period of the SCMFCs and both Toray paper and SGL followed the same trend, with H090-0 < H090-20 < H090-40 < H090-60 and S35-0 < S35-20 (Fig. 9).

The voltage trend of SCMFCs with different carbon materials was similar at the same PTFE content (H090-20 and S35-20) 


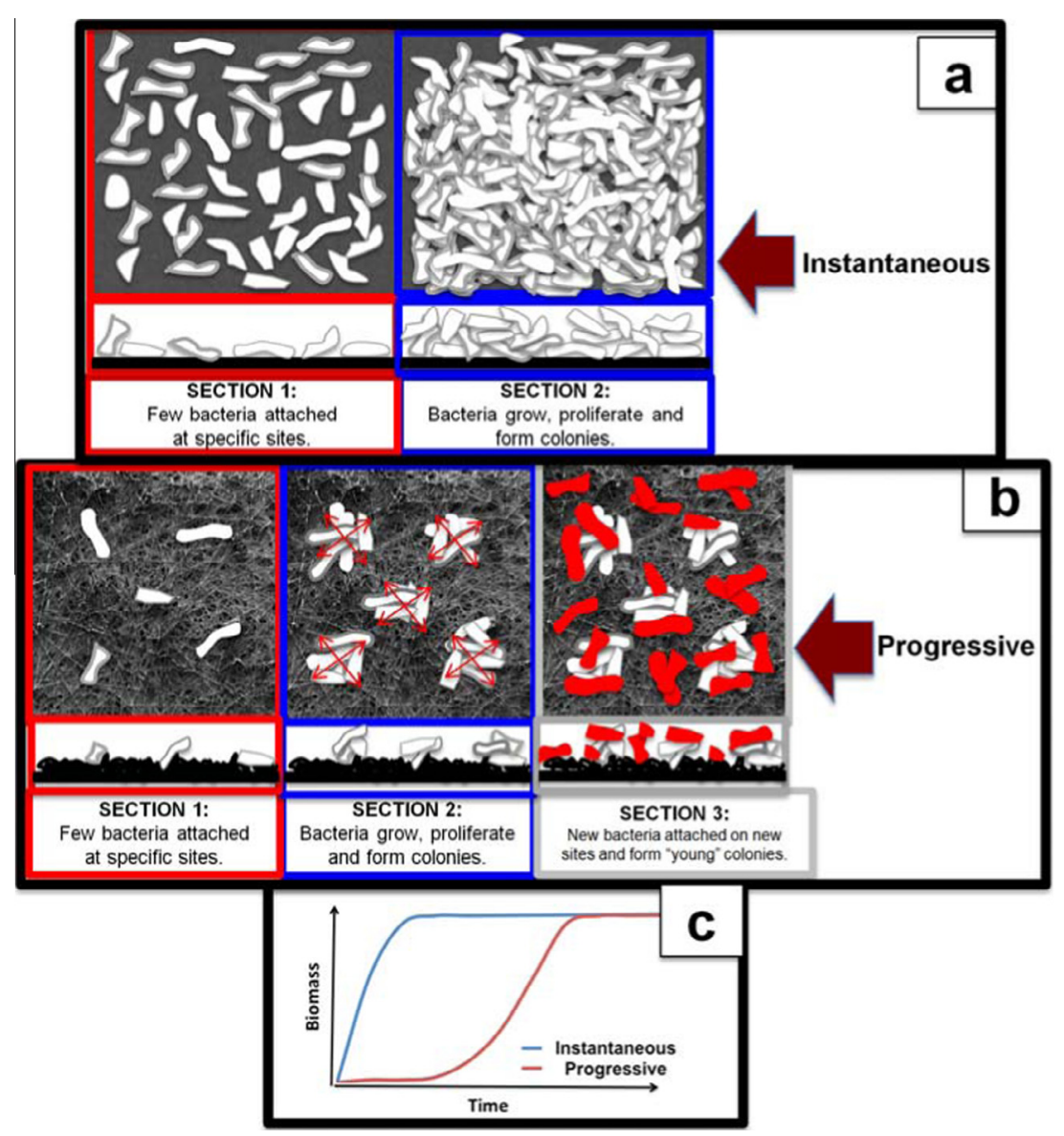

Fig. 7 - Schematic representation of proposed dynamics for biofilm formation and development (a) instantaneous and (b) progressive. Biomass variation in function of the dynamic proposed (c). (A colour version of this figure can be viewed online.)

(Fig. 9), which emphasized the relationship between PTFE content and the voltage generation during the start up time. The voltage difference between the carbon materials with different PTFE contents reduced over time. At the steady-state voltage, the SCMFCs with low/absent content of PTFE on the anode materials had roughly $20-30 \mathrm{mV}$ higher than those with high PTFE content ( $>40 \%$ ) (Fig. 9). Even if the start up time was faster with lower PTFE content, the PTFE content (up to $60 \mathrm{wt} \%$ ) slightly affected the performance of SCMFCs once the steady state voltage was reached. The slight difference in voltage was probably caused by the residual hydrophobicity of the carbon materials that slowed down the biofilm attachment onto the anode surfaces and by the higher ohmic resistance of carbon materials with higher PTFE content. This result was in agreement with the results of contact angle analysis where the hydrophobicity of carbon papers decreased substantially after the direct contact with wastewater (Fig. 3b).

\subsection{Electrode surface properties and the start up of MFCs}

The surface analysis gave a clear indication about the most suitable types of carbon material for anodes electrode to enhance the start up of MFCs. Low PTFE content (0-20 wt\% PTFE) in the carbon paper led to a substantial change from hydrophobic surface to hydrophilic surface after the direct contact with wastewater for 2 weeks. Moreover, the increase in the number of small pores $(5-10 \mu \mathrm{m})$ enhanced the biofilm attachment on the surface. The biofilm attachment could be enhanced by using more hydrophilic surfaces as shown for S35-0 and S35-20 carbon papers, which had a low resistance to water. TORAY H090-0 and H090-20 carbon papers also showed hydrophilic characteristics after 2 weeks in wastewater. In addition, the absence of a hydrophobic agent on the electrodes enhances the conductivity and thus facilitates the electron transfer in MFCs [1-3]. The MFCs with those materials as anode electrodes had a faster start up time.

On the other hand, higher PTFE content (40-60 wt\% PTFE, H090-40 and H090-60) provided surfaces that still showed slight hydrophobic properties even after biofilms started to grow on them. As the contact angle decreased after exposure to wastewater for these surfaces too, such slight hydrophobicity is then likely to disappear in long terms operation. The start up time of MFCs with higher PTFE content was longer than those with lower PTFE content, but after a few cycles the performances of MFCs were similar.

Concerning the use of agents to enhance the hydrophobicity of the cathode materials in MFCs, three points should be underlined. First, the hydrophobicity of the cathode in MFCs is important, since it would enhance the oxygen mass transfer to the three-phase (air-water-solid surface) inter- 


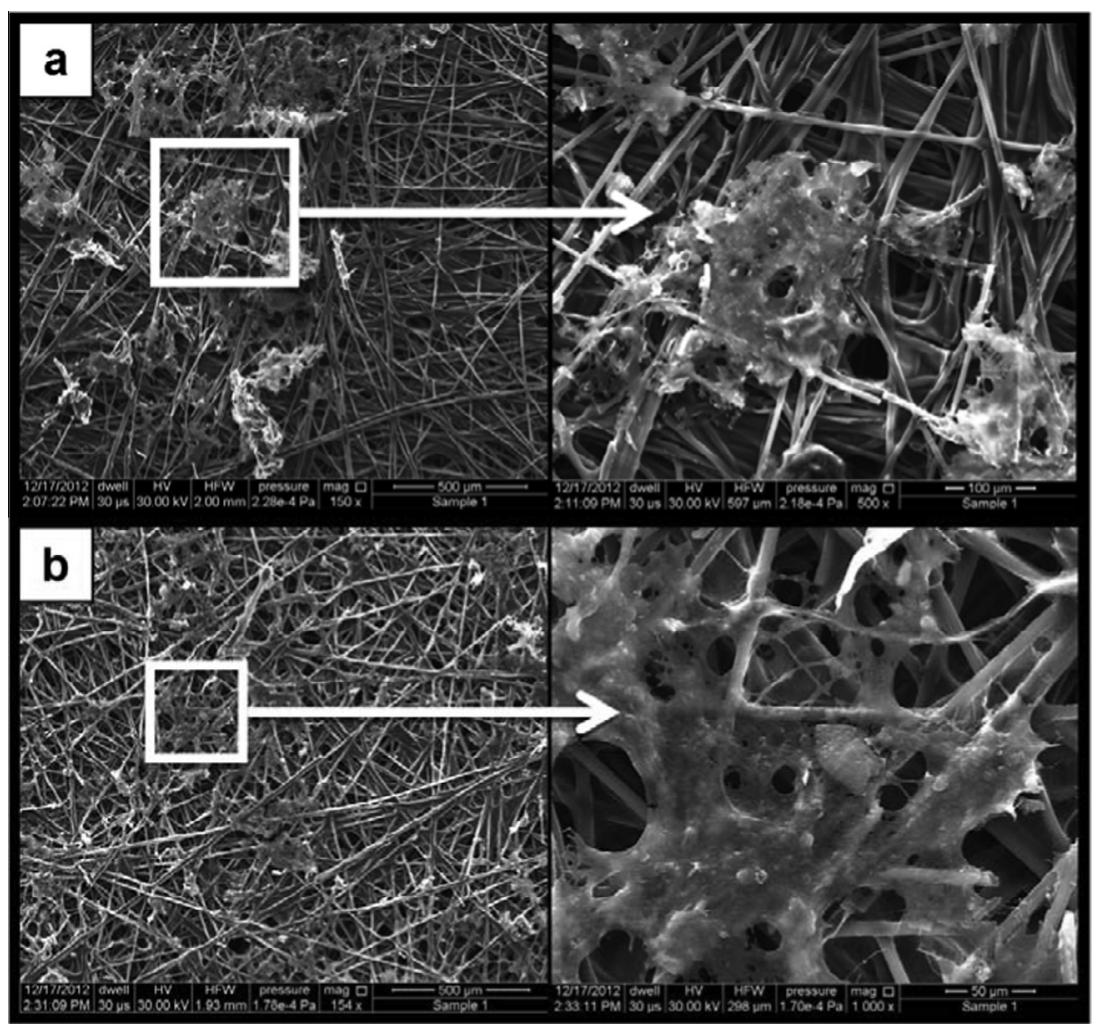

Fig. 8 - SEM images of H090-20 (a) and H090-60 sample (b) after biofilm growth.
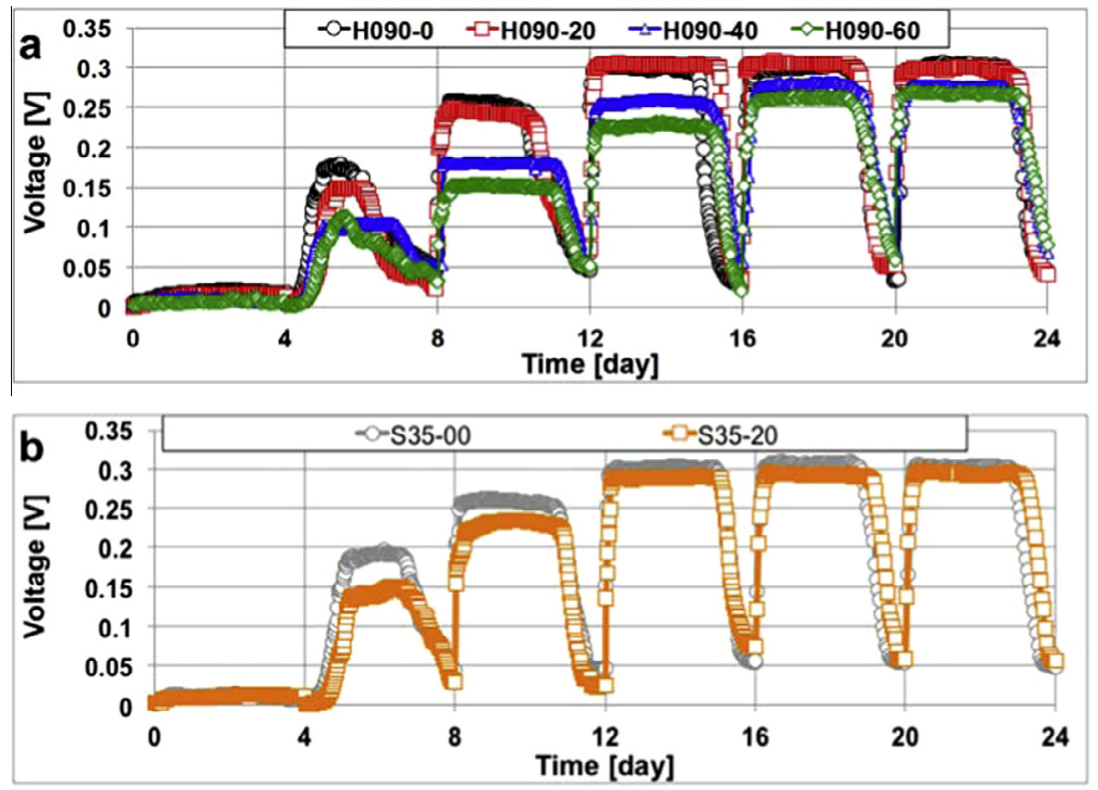

Fig. 9 - Start up voltage in SMFC using TORAY (a) and SGL (b) carbon papers. (A colour version of this figure can be viewed online.)

face at the reaction sites and consequently reduce the concentration polarization. Second, hydrophobic surfaces could slow down the biofilm growth on cathodes, which could prolong the lifetime of the cathode catalyst in MFCs [2,6]. This study clearly showed the change in hydrophobic characteristics of the materials surface after biofilm growth.
Third, the biofouling problem on cathodes was not completely solved especially for long-term operations as previously showed [42]. Other strategies, such as novel anti-fouling materials or MFC configurations should be developed to avoid biofouling, especially when expensive catalysts (i.e. Pt) are used. The utilization of biofilms as 
low-cost and reliable bio-cathode could also be an option for prolonging the lifetime of cathodes [43].

\section{Conclusions}

The surface properties (roughness, porosity and surface wettability) of the PTFE-treated carbon papers were extensively analysed, the association of the surface hydrophilicity/hydrophobicity and biofilm formation was determined, and the effects of electrode surface properties on the start up of MFCs were then investigated. The results showed a positive correlation between the amount of bacteria attached (dry biomass) and the surface porosity of the carbon papers at the small scale $(5-10 \mu \mathrm{m})$. The formation of biofilms on the carbon papers led to a significant decrease in the contact angle. The carbon papers with none or low PTFE treatment even changed to complete hydrophilic surfaces after contacting with wastewater for 2 weeks. Therefore, those materials should be applicable as anodes in MFC systems for shortening the start up time. In contrast, the carbon papers with high PTFE content maintained a light level of hydrophobicity after 2 weeks, indicating that the residual hydrophobicity along with the lower number of small pores $(5-10 \mu \mathrm{m})$ slowed down the biofilm attachment, and made the start up period of MFCs longer. After a few cycles, the voltage trend of MFCs with carbon papers with high PTFE content became similar to those with low PTFE content. The variation of the "wet" weight of the carbon papers over time indicated biofilm formation through progressive bacterial attachment.

\section{Acknowledgement}

Carlo Santoro was supported by the Electrochemical Society F.M. Becket Summer Fellowship 2013.

\section{R E F E R E N C E S}

[1] Rinaldi A, Mecheri B, Garavaglia V, Licoccia S, Di Nardo P, Traversa E. Engineering materials and biology to boost performance of microbial fuel cells: a critical review. Energy Environ Sci 2009;1:417-29.

[2] Wei J, Liang P, Huang X. Recent progress in electrodes for microbial fuel cells. Bioresour Technol 2011;102:9335-44.

[3] Karra U, Manickam SS, McCutcheon JR, Patel N, Li B. Power generation and organics removal from wastewater using activated carbon nanofiber (ACNF) microbial fuel cells (MFCs). Int J Hydrogen Energy 2013;3:1588-97.

[4] Carmona-Martinez AA, Harnisch F, Kuhlicke J, Neu TR, Schroeder U. Electron transfer and biofilm formation of Shewanella putrefaciens as function of anode potential. Bioelectrochem 2012. http://dx.doi.org/10.1016/ j.bioelechem.2012.05.002.

[5] Mathias MF, Roth J, Fleming J, Lehnert W, Vielstich W, Gasteiger HA, et al. Handbook of fuel cells fundamentals, technology and applications, vol. 3. New York: John Wiley \& Sons; 2003 [chapter 42].

[6] Santoro C, Agrios A, Pasaogullari U, Li B. Effects of gas diffusion layer (GDL) and micro porous layer (MPL) on cathode performance in microbial fuel cells (MFCs). Int J Hydrogen Energy 2011;36:13096-104.
[7] Young T. An essay on the cohesion of fluids. Philos Trans R Soc Lond 1805;95:65-87.

[8] Santini M, Guilizzoni M, Fest-Santini S. X-ray computed microtomography for drop shape analysis and contact angle measurement. J. Colloid Interface Sci. 2013;409:204-10.

[9] Gurau V, Bluemle MJ, De Castro ES, Tsou Y-M, Mann Jr JA, Zawodzinski Jr TA. Characterization of transport properties in gas diffusion layers for proton exchange membrane fuel cells: 1. Wettability (internal contact angle to water and surface energy of GDL fibers). J Power Sour 2006;160:1156-62.

[10] Santoro C, Stadlhofer A, Hacker V, Squadrito G, Schröder U, Li B. Activated carbon nanofibers (ACNF) as cathode for single chamber microbial fuel cells (SCMFCs). J Power Sour 2013;243:499-507.

[11] Ledezma P, Greenman J, Ieropoulos I. Maximising electricity production by controlling the biofilm specific growth rate in microbial fuel cells. Bioresour Technol 2012;118:615-8.

[12] Artyushkova K. GUI for calculating 1st and 2nd order statistics from images with Matlab, 2008.

[13] MathWorks, Natick, Massachusetts PLS toolbox 5.0 eigenvector research. Wenatchee, WA.

[14] Artyushkova K, Pylypenko S, Dowlapalli M, Atanassov P. Use of digital image processing of microscopic images and multivariate analysis for quantitative correlation of morphology, activity and durability of electrocatalysts. RSC Adv 2012;2(10):4304-10.

[15] Tadmor R, Yadav PS. As-placed contact angles for sessile drops. J Colloid Interface Sci 2008;317:241-6.

[16] Hung Y-L, Chang YY, Wang M-Y, Lin S-Y. A simple method for measuring the superhydrophobic contact angle with high accuracy. Rev Sci Instrum 2010;81:065-105.

[17] Nixon MS, Aguado AS. Feature extraction and image processing. 2nd ed. Academic Press; 2008.

[18] Del Rio OI, Neumann AW. Axisymmetric drop shape analysis: computational methods for the measurement of interfacial properties from the shape and dimensions of pendant and sessile drops. J Colloid Interface Sci 1997;196(2):136-47.

[19] Mack GL, Lee DA. The determination of contact angles from measurements of the dimensions of small bubbles and drops. II. the sessile drop method for obtuse angles. J Phys Chem 1936;40:169-76.

[20] Rotenberg Y, Boruvka L, Neumann AW. Determination of surface tension and contact angle from the shapes of axisymmetric fluid interfaces. J Colloid Interface Sci 1983;93:169-83.

[21] Gallo Stampino P, Balzarotti R, Cristiani C, Dotelli G, Guilizzoni M, Latorrata S. Effect of different hydrophobic agents onto the surface of gas diffusion layers for PEM-FC. Chem Eng Trans 2013;32:1603-8.

[22] Guilizzoni M, Gallo Stampino P, Cristiani C, Dotelli G, Latorrata S. Formulation and properties of different microporous layers with carboxymethylcellulose (CMC) composition for PEM-FC. Chem Eng Trans 2013;32:1657-62.

[23] DeBoor C. A practical guide to splines - revised edition, applied mathematical sciences, vol. 27. Springer; 1994.

[24] Bateni A, Susnar SS, Amirfazli A, Neumann AW. A highaccuracy polynomial fitting approach to determine contact angles. Colloids Surf 2003;A219:215-31.

[25] Guilizzoni M. Drop shape visualization and contact angle measurement on curved surfaces. J Colloid Interface Sci 2011;364:230-6.

[26] Santoro C, Ieropoulos I, Greenman J, Cristiani P, Vadas T, Mackay A, et al. Current generation in membraneless single chamber microbial fuel cells (MFCs) treating urine. J Power Sour 2013;238:190-6.

[27] Santoro C, Lei Y, Li B, Cristiani P. Power generation from wastewater using single chamber microbial fuel cells (MFCs) 
with platinum-free cathodes and pre-colonized anodes. Biochem Eng J 2012;62:8-16.

[28] Santoro C, Li B, Cristiani P, Squadrito G. Power generation of microbial fuel cells (MFCs) with low cathodic platinum loading. Int J Hydrogen Energy 2013;38:692-700.

[29] Gallo Stampino P, Cristiani C, Dotelli G, Omati L, Zampori L, Pelosato R, et al. Effect of different substrates, inks composition and rheology on coating deposition of microporous layer (MPL) for PEM-FCs. Catal Today 2009;147S:S30-5.

[30] Selvakumar N, Barshilia HC, Rajam KS. Effect of substrate roughness on the apparent surface free energy of sputter deposited superhydrophobic polytetrafluoroethylene coatings: a comparison of experimental data with different theoretical models. J Appl Phys 2010;108:013505-14.

[31] Miller JD, Veeramasuneni S, Drelich J, Yalamanchili MR. Effect of roughness as determined by atomic force microscopy on the wetting properties of PTFE thin films. Polym Eng Sci 1996;36(14):1849-55.

[32] Tadmor R. Approaches in wetting phenomena. Soft Matter 2011;7:1577-80.

[33] Wang T, Hu X, Dong S. A general route to transform normal hydrophilic cloths into superhydrophobic surfaces. Chem Commun 2007;2:1849-51.

[34] Hoefnagels HF, Wu D, de With G, Ming W. Biomimetic superhydrophobic and highly oleophobic cotton textiles. Langmuir 2007;23:13158-63.
[35] Cassie ABD, Baxter S. Wettability of porous surfaces. Trans Faraday Soc 1944;40:546-51.

[36] Moulinet S, Bartolo D. Life and death of a fakir droplet: impalement transitions on superhydrophobic surfaces. Eur Phys J 2007;E24:251-60.

[37] Wenzel RN. Resistance of solid surfaces to wetting by water. Ind Eng Chem 1936;28:988-94.

[38] Butt HJ, Graf K, Kappl M. Physics and chemistry at interfaces. Weinheim: Wiley-VCH; 2003. p. 127.

[39] Milchev A, Krastev I. Two-dimensional progressive and instantaneous nucleation with overlap: the case of multistep electrochemical reactions. Electrochim Acta 2011;56:2399-403.

[40] Milchev A. Electrocrystallization, nucleation and growth of nano-clusters on solid surfaces. Russ J Electrochem 2008;44(6):619-45.

[41] Vladkova T. Surface modification approach to control biofouling. Marine and industrial biofouling, vol. 4. Springer Series on Biofilm; 2009.

[42] Santoro C, Cremins M, Mackay A, Pasaogullari U, Guilizzoni M, Casalegno A, et al. Evaluation of water transport and oxygen presence in single chamber microbial fuel cells with carbon-based cathodes. J Electrochem Soc 2013;160(7):G128-34.

[43] Cristiani P, Carvalho ML, Guerrini E, Daghio M, Santoro C, Li B. Cathodic and anodic biofilms in single chamber microbial fuel cells. Bioelectrochem 2013;92:6-13. 